NYU-TH/12/98/01, CLNS 98/1600

\title{
Brane Inflation
}

\author{
Gia Dvali ${ }^{a}$ and S.-H. Henry Tye ${ }^{b}$ \\ ${ }^{a}$ Physics Department, New York University, 4 Washington Place, New York, NY 10003 \\ and ICTP, Trieste, Italy \\ ${ }^{b}$ Newman Laboratory of Nuclear Studies, Cornell University, Ithaca, NY 14853
}

(February 1, 2008)

\begin{abstract}
We present a novel inflationary scenario in theories with low scale $(\mathrm{TeV})$ quantum gravity, in which the standard model particles are localized on the branes whereas gravity propagates in the bulk of large extra dimensions. This inflationary scenario is natural in the brane world picture. In the lowest energy state, a number of branes sit on top of each other (or at an orientifold plane), so the vacuum energy cancels out. In the cosmological setting, some of the branes "start out" relatively displaced in the extra dimensions and the resulting vacuum energy triggers the exponential growth of the 3 non-compact dimensions. The number of e-foldings can be very large due to the very weak brane-brane interaction at large distances. In the effective four-dimensional field theory, the brane motion is described by a slowly rolling scalar field with an extremely flat plateau potential. When branes approach each other to a critical distance, the potential becomes steep and inflation ends rapidly. Then the branes "collide" and oscillate about the equilibrium point, releasing energy mostly into radiation on the branes.
\end{abstract}




\section{A. Introduction.}

Recently it was suggested that the fundamental scale of quantum gravity $M_{P f}$ may be as low as TeV, providing an alternative understanding of the hierarchy problem [1]. Observed weakness of gravity at large distances is associated by $N$ large new dimensions (of size $\left.\sim R>>M_{P f}^{-1}\right)$ in which gravity can propagate. Then the relation between the observed Planck scale $M_{P}$ and the fundamental Planck scale $M_{P f}$ is given by

$$
M_{P}^{2}=M_{P f}^{N+2} V_{N}
$$

where $V_{N} \sim R^{N}$ ( $N$ must be 2 or larger) is the volume in extra spatial dimensions (i.e., the bulk). In this picture, all the standard model particles must live in a brane (or a set of branes) with 3 extended space dimensions [1] picture is in the string context via the $D$-brane construction (see [6] for an introduction), where the standard model fields can be identified with open string modes stuck on the $D$-branes and the graviton is a closed string mode propagating in the bulk [7-10].

Various phenomenological and astrophysical constraints [1] and related issues like black holes [11], collider physics [12], flavor violation [13], neutrino masses [14, 15] and proton decay [1,15] have been addressed. Unification of the gauge couplings is another important issue and in this respect, the proposals of [16], [15] look particularly promising.

The main concern of the present paper is the inflationary cosmology in this framework. Obviously, with the above proposal, the standard cosmological picture is dramatically affected. As it was shown in [1], big bang nucleosynthesis (BBN) and the bulk graviton production place severe bounds both on the reheating temperature and on the "initial conditions" for the hot big bang after inflation. First, the reheat temperature must be above at least $1 \mathrm{MeV}$ for BBN to proceed successfully. On the other hand too much reheating in the bulk can lead to the overproduction of Kaluza-Klein (KK) gravitons which can over-close the Universe and ruin the success of BBN. Therefore the minimal requirement after inflation would be to reach a reasonable reheating temperature on the brane $T_{R}$, while the bulk remains cold and empty|2. The question is how to implement inflation in this scenario to achieve these properties, in addition to sufficient inflation, and correct amounts of density perturbation and baryogenesis? Some potential problems in this respect were considered in [17] [18]. Their considerations, however, were limited to conventional inflation potential with the inflaton being one of the fields living on the brane. This type of scenarios typically requires tremendous fine tuning.

In this letter we suggest a novel inflationary scenario, free of the above difficulties. The crucial aspect is that, although the inflaton is a brane mode in the ground state, it behaves as an "inter-brane" mode that describes a relative separation of branes in the extra space

\footnotetext{
${ }^{1}$ In a different context an attempt of lowering the string scale to $\mathrm{TeV}$, without lowering the fundamental Planck scale was considered in [2], based on an earlier observation in [3]. Dynamical localization of the fields on a (solitonic) brane embedded in a higher dimensional universe has been studied earlier in the field theoretic context for spin-0 and 1/2 [4], [5] and spin-1 states [5]

${ }^{2}$ For recent discussions on thermal phase transitions in higher dimensional theories see [19], [20].
} 
directions. This separation induces inflation in the 3 non-compact dimensions. During inflation, when branes are generically separated, the inflaton behaves as a weakly coupled field (as it should), with a slow-roll behavior. Towards the end of inflation, when branes come close, the inflaton couples to the other brane modes but remains very weakly coupled to the bulk modes. This means only the brane is reheated, thus avoiding the problems with late entropy release or over-closure of the Universe. For the outside observer, this process would look like reheating due to the "breathing" of brane-brane bound-state. An oversimplied inflaton potential that illustrates the above generical feature has the form:

$$
V(\phi) \sim M_{P f}^{4} \xi\left(\phi / M_{P f}\right)\left(1-e^{(-|\phi| / m)}\right)
$$

where $m$ is a model-dependent mass scale and $\xi(\phi)$ is a smooth slowly-varying function.

This inflationary scenario emerges rather naturally in the generic brane world picture [1,10]. We may consider the Type I string where $K$ branes sit more or less on top of an orientifold plane at the lowest energy state, resulting in zero cosmological constant. In the cosmological setting, it is reasonable to assume that some of the branes were relatively displaced from the orientifold plane in the early universe. This displacement induces an effective vacuum energy density triggering inflation. The separation distance may be identified with the vacuum expectation value $\phi$ of an appropriate Higgs field [21. This Higgs field is an open string state with its two ends stuck on two separated branes; that is, this scalar field is a brane mode playing the role of the inflaton. In the effective four-dimensional theory, the motion of the branes is described by this slowly-rolling scalar field, the inflaton. This has a flat potential with no need of fine tuning. Flatness of its potential is due to the very weak brane-brane (and brane-orientifold plane) force at large distances, thus allowing for sufficient number of e-foldings. When the branes come closer, the short-range potential turns on and ends inflation. At this point the inflaton potential becomes very steep and its oscillations reheat the brane(s). In this scenario, it is even possible that the electroweak Higgs field plays the role of the inflaton.

\section{B. Brane-induced inflation.}

In this subsection we show how the relative separation of the branes in the extra space can lead to the inflation in 3 non-compact dimensions. Consider two 3-branes" 2 . We will be assuming that there is an attractive potential between these branes which stabilizes them on top of each other. Let the brane tension, the energy per unit 3 -volume, be $T$ and the brane-brane separation in the extra space be $r$. Then the effective 4-dimensional vacuum energy density is given by

$$
\Lambda_{\text {eff }}=V(r)+2 T+\Lambda_{b u l k} V_{N}
$$

where $V(r)$ is the brane-brane interaction energy and $\Lambda_{b u l k}$ is an effective bulk cosmological constant. Their normalization is such that $V(0)=0$ and the positive contribution from

\footnotetext{
${ }^{3}$ one can in fact consider them as two $p$-branes with $p-3$ dimensions compactified (see the next section).
} 
$T$ is canceled out by the negative contribution from $\Lambda_{b u l k}$. When branes are displaced, $V(r)$ is no longer zero and results in an effective cosmological constant in four dimensions $\Lambda_{\text {eff }}=V(r)>0$. What is the cosmological effect of this term? Before discussing this, let us make a couple of clarifying remarks.

To preserve the success of $\mathrm{BBN}$, there is a very strong requirement that the size of the extra dimensions should not evolve from the time of nucleosynthesis. On the other hand, for small $N$, it is not desirable to have a large reheating temperature in the bulk, since the resulting KK graviton excitations can over-close the universe. In the present context, this is quite natural since the reheating takes place on the brane(s) only (see below), so we expect little change in the bulk temperature in this scenario after inflation. Therefore, in the first approximation, we require that extra radii are frozen both during and after inflation. For this to be the case, the radius modulus (radion) must be stabilized by some effective potentialf which can give it a mass $m_{R}$ larger than an effective four-dimensional Hubble parameter

$$
m_{R}>H=\left(\Lambda_{e f f} / 3 M_{P}^{2}\right)^{\frac{1}{2}}
$$

This is not a very strong requirement since we expect at most $\Lambda_{\text {eff }}=V(r) \sim M_{P f}^{4}$.

The second point is that an effective 4-dimensional Hubble size $H^{-1}$ should be larger than the size of extra dimensions $R$. Otherwise the universe cannot be treated as four dimensional at distances $\sim H^{-1}$. In view of (3), this is equivalent to the requirement that the $(4+N)$-dimensional Bulk Hubble length

$$
H_{b u l k}^{-1} \sim \frac{M_{P f}^{1+N / 2}}{\Lambda_{b u l k}^{1 / 2}}>R
$$

Both of these requirements are easily satisfied for $\Lambda_{e f f} \sim T \sim M_{P f}^{4}$, even for $N=2$.

Assuming that the above requirements are satisfied, at distances $>>R$ the evolution of the 4-dimensional scale factor $a(t)$ is governed by the usual Friedmann expansion, with an effective Hubble parameter

$$
H^{2}=\left(\frac{\dot{a}}{a}\right)^{2}=\frac{\rho_{e f f}}{3 M_{P}^{2}}
$$

where dot denotes a derivative with respect to the cosmic time $t$ and

$$
\rho_{\text {eff }}=T \dot{r}^{2}+V(r)
$$

is an effective four-dimensional energy density of the Universe. The first term in (7) comes from the kinetic energy of relative brane motion (we neglect the center-of-mass motion). Inflation will take place if

\footnotetext{
${ }^{4}$ We will not try to identify an explicit source of the radius stabilization in this paper and will take it for granted (see [22], [23] for possibilities in this respect and [24] for recent discussions on perturbative stability ).
} 


$$
T \dot{r}^{2}<<V(r)
$$

meaning that $V(r)$ is a flat function for some $r$. On the other hand, effective reheating requires $V(r)$ to be steeper at the later stages. This is the key point of our paper: in many cases, $V(r)$ is indeed a steep function for small $r$ rapidly approaching a constant value for $r \rightarrow \infty$. To see this, consider the brane-brane interaction at large distances. It is governed by the following possible sources:

1) There are modes (both massive and massless) localized on the branes. Their wavefunction decays exponentially fast in the directions transverse to the brane $\psi(r) \sim e^{-r / r_{0}}$ where $r_{0}$ is the "thickness" of a brane, an effective localization width of the states. Many of the standard model particles belong to this category. When branes are separated, the couplings between the states from different branes are suppressed. When the branes are coincident, however, the coupling strengths are restored and they contribute to the vacuum energy on the brane. Apriori this contribution cannot vanish since supersymmetry must be broken on the brane, where we live. Its sign depends on the details. We assume that this contribution is negative (in agreement with the assumed attractive brane-brane potential) and thus must be canceled out by adjusting a positive brane tension. This is just the usual fine tuning of the cosmological constant. On the other hand, when branes are separated, the negative contribution decreases (at least) exponentially fast $\sim-e^{-r / r_{0}}$ and there must remain a non-balanced positive constant term at large distances. The bottom-line is that, there is a very short range attractive potential between the branes:

$$
V(r) \sim T\left(1-f\left(r / r_{0}\right)\right)
$$

where $f\left(r / r_{0}\right)$ is an exponentially vanishing function for $r>r_{0}$. For solitonic membranes we expect $r_{0}$ to be at least as small as $T^{-\frac{1}{4}} \sim M_{P f}^{-1}$.

2) Another potential source is an exchange of the massive bulk modes, which at large distances $\left(r>>m^{-1}\right)$ decouple and create an Yukawa suppressed potential $\sim r^{2-N} e^{-m r}$.

3) In the same way, an exchange of the light bulk modes, like graviton or gauge fields will generate a power-law potential $\sim 1 / r^{N-2}$; an over-all sign depends on the balance between the tension and gauge charge density on the brane $e^{\varpi}$. For instance, gravitational interaction is attractive between $D$-branes and repulsive between $D$-branes and the orientifold plane, while the R-R fields have an opposite effect and in the unbroken supersymmetry limit can compensate the graviton/dilaton force. In this limit, branes are BPS states. However, even if the RR fields and the dilaton field get small masses, their effect can still "screen" gravity at distances $r<<m^{-1}$.

4) Confining potential $\sim k r$ due to the strings stretching between the branes. One expects open strings stretched between branes. This is true both for $D$-branes [6] as well as for field theoretic solitons [5]. In the latter case they are flux tubes which are presented due to the fact that bulk must be confining (in order to have a massless gauge field localized on

\footnotetext{
${ }^{5}$ By "effective", we mean $T_{R}$ at least up to nucleosynthesis temperature $T_{R} \sim 1 \mathrm{MeV}$ on the brane.

${ }^{6}$ In some cases, $\log r$ dependence should be understood for the $N=2$ case.
} 
the brane) [5] and therefore the gauge flux is trapped in the flux tubes that can stretch from brane to brane. Thus, at large distances one expects the brane-brane potential to be

$$
V(r)=T\left(\alpha-f\left(r / r_{0}\right)+b_{i} \frac{e^{-m_{i} r}}{r^{N-2}}+\frac{c}{r^{N-2}}+k r\right)
$$

where $\alpha, b_{i}, c$ are model-dependent constants. $k$ is proportional to the density of the stretched strings per unit 3-volume. During inflation, $k$ is redshifted away exponentially fast $k(t) \sim$ $k_{i n} e^{-3 H t}$. The condition that the linear term will allow inflation is

$$
\frac{M_{P} k_{i n}}{M_{P f}^{2}\left(a T+k_{i n} r_{i n}\right)}<1
$$

at least in some region of $H^{-1}$ size. The corresponding region will then expand making $k$ negligible very rapidly]. In what follows we will assume that this is the case and ignore the linear term.

We now turn to the discussion of the resulting inflationary dynamics. It is most convenient to do this in the effective 4-dimensional field theory picture, where brane-brane separation is described by an expectation value of the real scalar field (inflaton):

$$
\phi \sim M_{P l}^{2} r
$$

There is a simple understanding of this relation (see the next section for an alternative explanation in the $D$-brane context) if one thinks of branes as some sort of the field theory solitons formed by the massive scalar fields $\Phi_{1}$ and $\Phi_{2}$ with the Lagrangian

$$
L=L_{1}\left(\Phi_{1}\right)+L_{2}\left(\Phi_{2}\right)+L_{\text {cross }}\left(\Phi_{1} \Phi_{2}\right)
$$

In this language branes are stable configurations independent of $x_{\mu}$ and localized in the external space at $x_{A}=0$ and $x_{A}=r_{A}$ respectively

$$
\Phi_{1}=\Phi_{1}\left(x_{A}\right), \quad \Phi_{2}=\Phi_{2}\left(x_{A}-r_{A}\right)
$$

(where the index $A=1,2 . ., N$ labels the extra coordinates). It is most crucial that away from the branes the situation looks like the translational invariant vacuum up to an exponentially small correction $\sim e^{-m|x|}$. Assume for a moment that there is no cross interaction among $\Phi_{1}$ and $\Phi_{2}$ fields in the Lagrangian $\left(L_{\text {cross }}=0\right)$. Then there are $N$ massless modes localized on each brane, corresponding to $N$ transverse excitations:

$$
\Phi_{i}\left(x_{A}+G_{A}^{i}\left(x_{\mu}\right)\right)=\text { brane }+G^{i}\left(x_{\mu}\right) \frac{\partial \Phi_{i}\left(x_{A}\right)}{\partial x_{A}}+\text { heavy modes }
$$

These are Goldstone modes of spontaneously broken translational invariance. However, in the presence of two branes only $N$ superpositions $\left(G_{A}^{1}+G_{A}^{2}\right)$, corresponding to the center

\footnotetext{
${ }^{7}$ Note that for the regions with $k \sim M_{P f}^{-1}$ inflation would require either $r_{i n} \sim M_{P} / M_{P f}^{2} \sim 1 \mathrm{~mm}$, or $T<M_{P f}$.
} 
of mass motion, are true Goldstones modes 8 . The remaining $N$ orthogonal combinations $\phi_{A}\left(x_{\mu}\right)=G_{A}^{1}-G_{A}^{2}$ correspond to relative vibrations of the branes and are massless only because membranes are non-interacting. However, even in the presence of local cross couplings, e.g.

$$
L_{\text {cross }}=\lambda\left(\Phi_{1} \Phi_{2}\right)^{2}
$$

the induced potential is short range, due to localized nature of branes, and decays exponentially fast for large separation. Corresponding four-dimensional mode describing this separation is given by

$$
\phi=\left(\left(r_{A}+G_{A}^{1}-G_{A}^{2}\right)^{2}\right)^{\frac{1}{2}}
$$

and has an exponentially decaying potential for large field values

$$
V(\phi) \sim T(\alpha-f(\phi / M))
$$

where $f(\phi / M)$ goes at least as $\sim e^{-\phi / M}$ and $M \sim T^{\frac{1}{4}}$ is a typical mass parameter of the theory. As discussed above, the constant term $\alpha$ comes from the short range binding energy of the solitons, which at the tree level is roughly:

$$
E_{\text {binding }} \sim \int d V_{N}\left(L_{\text {cross }}\left(\Phi_{1}\left(x_{A}\right) \Phi_{2}\left(x_{A}\right)\right)-L_{\text {cross }}\left(\Phi_{1}\left(x_{A}\right) \Phi_{2}(\infty)\right)\right)
$$

There will be an additional contribution to $\alpha$ and $f$ from the modes localized on the solitons. All this contributions are very short range bounded by the brane thickness. In the presence of light bulk modes, such as gravity or gauge fields, there will be an additional interaction of the form $(\sqrt{10})$ so that the effective potential will become:

$$
V(\phi) \sim T\left(\alpha-f(\phi / M)+\frac{1}{|\phi|^{N-2}}\left(\beta_{i} e^{-|\phi| / M_{i}}+\gamma\right)\right)
$$

where index $i$ runs over all the massive modes. Note that $M_{i} \sim M_{P f}^{2} / m_{i}$ depend on the masses of bulk modes and apriori may be totally unrelated to the value of $T$.

Let us now turn to the inflationary dynamics following from the above potential. We will assume that all the bulk masses are $m_{i}<M_{P f}$ and, therefore, for $\phi>>m_{i}^{-1}$ the short range potential $f(\phi / M)$ plays no role. Note that for $m_{i}<<R^{-1}$ case, it is possible that $\beta_{i}$ and $c$ terms conspire and cancel out for $r<<m_{i}^{-1}$. In such a case $V(r)$ can be dominated by $f(r)$ term. This may happen in the string theory and will be discussed later. For the moment however we assume that there is no such a conspiracy and will discuss the case of very small $\gamma$ first.

As mentioned above, we assume that Universe starts out in the state with nonzero vacuum energy induced by (parallel) branes separated at some distance $r_{i n} \sim \phi_{\text {in }}$ and

\footnotetext{
${ }^{8}$ In the presence of gravity, these are eaten up by graviphotons $g_{\mu A}$ and get mass $\sim T^{\frac{1}{4}} M_{P f} / M_{P}$ [1]
} 
slowly approaching each other under the action of an attractive force. In the effective fourdimensional language this is described by the slowly-rolling scalar field $\phi$, whose evolution is governed by the following equation of motion

$$
\frac{d^{2} \phi}{d t^{2}}+3 H \frac{d \phi}{d t}+\frac{d V}{d \phi}=0
$$

where $V$ is given by (20) and we take $\gamma=0$ to start with. Standard slow-roll conditions are

$$
\left|M_{P} V^{\prime} / V\right|<<1, \quad\left|M_{P}^{2} V^{\prime \prime} / V\right|<<1
$$

where prime denotes the derivative with respect to $\phi$. Breakdown of either of these will mark the end of inflation. The corresponding value $\phi_{\text {end }}$ is determined by:

$$
\frac{\beta}{\alpha \phi_{\text {end }}^{N-2}} e^{-\frac{\phi_{\text {end }}}{M}} \simeq\left(\frac{M}{M_{P}}\right)^{2}
$$

The number of $e$-foldings $n$ between two values $\phi_{\text {end }}$ and $\phi_{n}$ can be found by integrating (21) subject to the slow roll (basically ignoring the second derivative)

$$
n=\frac{1}{M_{P}^{2}} \int_{\phi_{\text {end }}}^{\phi_{n}} d \phi \frac{V}{d V / d \phi}
$$

This gives

$$
\frac{\beta}{\alpha \phi_{n}^{N-2}} e^{-\frac{\phi_{n}}{M}} \simeq\left(\frac{M}{M_{P}}\right)^{2} \frac{1}{(n+1)}
$$

Comparing with (23) we see that during the last 60 e-folds, the change in $\phi$ is very small. Thus for small $\gamma$ the requirement of successful inflation puts basically no constraint on the number and size of extra dimensions as far as $R>m_{i}^{-1}$. For $\gamma \sim \beta_{i}$ the slope of the potential at $\phi>>M_{i}$ is dominated by the power-law term and the slow-roll breaks down at

$$
\alpha \phi_{e n d}^{N}=\gamma M_{P}^{2}(N-1)(N-2)
$$

and the value $\phi_{n}$ ( $n$ e-folds before the end of inflation) is given by

$$
\alpha \phi_{n}^{N}=(n+1) \gamma M_{P}^{2} N(N-2)
$$

Now using equations (11) and (12) and taking into account that the maximal initial separation of the branes is bounded by the size of extra dimension $r_{i n}<R$, we get that for having 60 e-foldings with maximal initial separation, $c$ must satisfy

$$
M_{P f}^{N-2}>60(N-1)(N-2) \gamma / \alpha
$$

which is not a significant constraint. 


\section{The Type I String Picture}

The observed universe contains gauge and matter fields as well as gravity. Since superstring theory [6] is the only known theory that incorporates consistent quantum gravity, one would like to see how string theory can describe our universe. In the brane world picture [1.:4:10], gravity lives in the ten dimensional space-time, whereas gauge fields can be localized on $p$ spatial dimensional extended objects known as $D p$-branes. In particular, our four dimensional world (including the strong and electroweak interactions as well as the quarks and leptons) resides inside a set of overlapping branes (or intersection thereof with other branes), with the extra $p-3 \geq 0$ spatial dimensions compactified on a manifold with some finite volume $V_{p-3}$. However, gravity is free to propagate in the ten dimensional bulk of the space-time with the remaining $9-p$ spatial dimensions compactified on a manifold with some finite volume $V_{9-p}$. Dilaton (and other moduli) stabilization generically requires the string coupling to be $g_{s} \gtrsim 1$. Thus, in the brane world picture, the four dimensional gauge and gravitational couplings scale as $1 / V_{p-3}$ and $1 / V_{p-3} V_{9-p}$, respectively. By tuning $V_{9-p}$ (for $p<9$ ) we can achieve gauge and gravitational coupling unification. Then for $p>3$, we can choose $V_{p-3}$ large enough so that the four dimensional gauge couplings are small even if $g_{s}$ is not. The $3<p<9$ feature means that our world is inside a $p$-brane. In this brane world picture, the string scale $M_{s}$ can apriori be anywhere between the electroweak scale and the Planck scale. Physics becomes most exciting when $M_{s}$ is around TeVs [1]. Note that

$$
M_{P}^{2} \sim M_{s}^{8} V_{p-3} V_{9-p}
$$

Comparing with (11), we obtain the relation

$$
M_{P f}^{2+N} \sim M_{s}^{8} V_{p-3} V_{9-p-N}
$$

Here $M_{P f}$ is expected to be somewhat larger than $M_{s}$, but within the same order of magnitude.

To see that the inflaton potential during the inflationary epoch can appear rather naturally in the brane world, let us consider the embedding of the $\mathrm{TeV}$ scale scenario in the perturbative 4-dimensional Type I string [7,25], where some semi-realistic models have been constructed [8.91. A typical model will have orientifold planes with negative tensions (i.e., energy density) [26]. It turns out that the orientifold plane is also charged under some $(p+1$ form) tensor (i.e., RR) fields present in the theory. One may view these planes as rigid $p$-branes with negative brane tensions and negative $\mathrm{RR}$ charges. (To be concrete, one may choose $p=5$.) When all the $9-p$ directions are compactified, the negative $\mathrm{RR}$ charges of the orientifold plane must be canceled by the introduction of positively charged D-branes. In the classical vacuum of a Type I string, a set of D-branes sit on top of each orientifold plane, with zero net $\mathrm{RR}$ charge. Let the brane tension of a $\mathrm{D} p$-brane be $T_{p}$. Then the sum of the energy density $K T_{p}$ of the set of $K \mathrm{D} p$-branes exactly cancels the negative energy density $-K T_{p}$ of the orientifold plane $\left(K=2^{l}\right)$. In a supersymmetric vacuum, the energy density is exactly zero everywhere. (There are vacua in which the branes are separated. F theory [27] provides the proper description of these generalized situations.)

In the early universe, however, the $\mathrm{D} p$-branes do not have to be exactly on top of the orientifold plane. Let us consider the simple situation (this is also the most relevant situation, 
as we shall explain later) where a single $\mathrm{D} p$-branes is separated from the rest by a distance $r$. The tension (energy density) of the brane, $T_{p} \sim M_{s}^{p+1}$, is opposite to the sum of the enrgy density of the remaining branes plus the orientifold plane. Before dilaton (and other moduli) stabilization and SUSY breaking, the static potential due to the exchange of the closed string sector fields has the form [28]

$$
V(r) \sim T_{p}(1-1) F(r)
$$

where $r$ is the displacement distance in the extra large directions, and the function $F(r)$ is model dependent. Crudely speaking, $F(r)$ is the dual of the one-loop open string amplitude. This potential arises from the exchange of closed string (bulk) states between the displaced $\mathrm{D} p$-brane and the rest. The closed string spectrum can be separated into two groups, the NS-NS states and the RR states. The positive term comes from the exchange of NS-NS states and the negative terms come from the exchange of the RR states. The massless NSNS states include the graviton and the dilaton-axion. The force due to their exchanges is repulsive. The exact cancellation of the forces due the NS-NS and RR fields is a consequence of the BPS property of the branes. If the brane is moving slowly towards the rest, velocitydependent terms are expected in the potential. Before supersymmetry breaking, the leading term has the form $v^{4} r^{p-7}$. After supersymmetry breaking, we expect a $v^{2}$ term to be present.

In the more realistic situation, where the string model describes our universe, the dilaton (and other moduli) must be stabilized dynamically, and supersymmtry must be broken (presumably softly and dynamically). To be compatible with experiments, all the bosonic bulk modes except the graviton are expected to become massive. This means that the dilaton-axion and the RR fields must become massive (so that their long range forces are Yukawa suppressed), while only gravity remains long ranged. After the stabilization of the dilaton and other moduli, we expect the potential $V(r)$ to be, at large $r$,

$$
V(r)=M_{P f}^{6-N} r^{2-N}\left(1+\sum e^{\left(-m_{i} r\right)}-\sum e^{\left(-m_{j}^{\prime} r\right)}\right)
$$

where $m_{i}$ are the masses of the NS-NS states while $m_{j}^{\prime}$ are the masses of the RR fields. For large $r$ and $\mathrm{N}=2, V(r)$ is essentially a constant. For $r=0$, we expect $V(r=0)=0$, since today's cosmological constant is (almost) zero. For small $r$, the form of $V(r)$ depends crucially on the mass spectrum. However, the overall shape of $V(r)$ is quite clear: it rises rapidly from zero at $r=0$ to the $r^{2-N}$ form for large $r$.

Besides the massive states (i.e., $\sim M_{P f}$ or larger) which we may safely ignore, there are bulk light states which were massless before supersymmetry breaking and dilaton stabilization. For experimental compatibility, it is enough that their masses are of the order of inverse millimeter $(\mathrm{mm})$ or larger. (From the experimental view-point, this is a very exciting scenario, due to the sub-millimeter test of Newton's gravitational law [29]). This will automatically be the case if the only stabilizing potential for them comes from supersymmetry breaking on the brane [7]. In this case, of course, their contributions will keep canceling gravitation at distances smaller than inverse mm. Effectively, the coefficient of $1 / r^{N-2}$ term ( $c$ in the previous section) will be suppressed at least by a factor $\sim \frac{M_{P f}^{2}}{M_{P}}$, since this measures the transmission of the supersymmetry breaking in the brane to the bulk and thus generates the dilaton-axion-RR masses. So the number of e-foldings can be very large! In general, the possible number of e-foldings will be bounded by Eq.(27). 
In the $\mathrm{TeV}$ scenario, supersymmetry must be broken on the brane in full strength, so we expect the appearance of the constant term in (32), of the form $T \alpha$, which should come from the short-range brane-brane binding energy, an analog of (19), as well as Higgs potential from the brane modes.

Now we want to argue that the separation distance $r$ is really a brane mode, not a bulk mode. This is crucial to the reheating problem. Suppose the displaced brane belongs to a set of branes that yield a $S U(L)$ gauge symmetry, i.e., the $S U(L)$ gauge bosons are open strings whose ends live on this set of branes. Then the separation of the branes breaks the $S U(L)$ gauge symmetry, resulting in some massive gauge bosons [21]. The energy due to a stretched open string is $M_{s}^{2} r$ (i.e., string tension times length). However, we know that this corresponds to the mass of a gauge boson after spontaneous symmetry breaking,

$$
\text { mass }=g \phi \sim M_{s}^{2} r
$$

so we can identify $r$ with the vev of the Higgs field $\phi$. Here $\phi$ is an open string state whose one end is stuck on the displaced brane, while its other end is stuck on one (or an appropriate linear combination) of branes that are sitting on the orientifold plane. So it is a brane mode. (At the ground state, $\phi$ lives mostly inside the branes.) After the integration over the extra large dimensions, the above potential $V(r)$ becomes the potential term $V(\phi)$ in the 4-dimensional effective field theory, so $\phi$ becomes the inflaton. More generally, even when there is no gauge symmetry associated with the separation of a particular brane, the separation distance $r$ is identified with the vacuum expectation value of an appropriate scalar field; that is, this scalar field is a brane mode playing the role of the inflaton. In the effective four-dimensional theory, the motion of the branes is described by this slowly-rolling scalar field, the inflaton. This has a flat potential with no need of fine tuning.

In the early universe, some number of open strings are stretched between the branes. The density of such stretched strings (or other stretched branes) depends on the initial condition/situation. This density will decrease as the universe expands. In fact, inflation will shift it to zero rapidly.

At $\phi=0$, we know $V(\phi=0)=0$, so that the cosmological constant remains zero. At small $\phi$, we know the potential is quite steep. In fact, the precise shape is very model/dynamics dependent. Fortunately, the details there are not so important. Since $\phi$ is a brane mode, its coupling to closed string (i.e., bulk) modes are extremely weak, while its coupling to other open string (i.e., brane) modes are around typical gauge coupling strength, so its damping reheats predominantly the brane but not the bulk.

In this brane inflation scenario, the inflaton could have been the electroweak Higgs field in the standard model. In this case, the minimum of the potential should be shifted away from the $\phi=0$ point to the electroweak scale. This can be achieved if the electroweak Higgs potential is generated after supersymmetry breaking.

Now we can consider the general case, where the branes are all separated from the orientifold plane. The separations may even be at different angles. A careful treatment requires an analysis in $\mathrm{F}$ theory [27]. However, we can easily envision that, one after another, the branes begin falling on top of the orientifold, until only one brane, or a set of overlapping branes, remains separated from the rest. As long as this separation survives 60 e-foldings, the details before that is irrelevant to observations today. 


\section{Density Perturbation and Baryogenesis.}

Now let us discuss the issue of density perturbations in our scenario. The predicted value of $\frac{\delta \rho}{\rho}$ can be estimated from the standard formula

$$
\frac{\delta \rho}{\rho} \sim \frac{H}{M_{P} \epsilon}
$$

where $\epsilon=M_{P} V^{\prime} / V$ is the slow-roll parameter in (22). The right-hand side of the above equation must be evaluated at a time when the scale of interest crosses out of the de Sitter horizon $H^{-1}$. Let the corresponding inflaton value be $\phi_{n}$. Perturbations on the present Hubble scales correspond to the value of $\frac{\delta \rho}{\rho}$ at $\phi=\phi_{60}$. In the usual picture (with $M_{P f} \sim$ $\left.M_{P}\right)$ the Hubble parameter can be reasonably large $\left(\sim 10^{13}-10^{14} \mathrm{GeV}\right.$ or so $)$ and therefore the observed value of $\frac{\delta \rho}{\rho} \sim 10^{-5}$ does not require very small $\epsilon_{60}$. In our case the Hubble

parameter is tiny $\frac{H}{M_{P}} \sim 10^{-32}$ and thus $\epsilon_{60}$ must be enormously small $\left(10^{-28}\right.$ or so!). This means that the potential at $\phi_{60}$ must be extremely flat and sharply become very steep at $\phi_{\text {end }}$. This can be achieved if the inflaton potential is dominated by the short range brane-brane interaction. Very crudely, the density perturbations then come out to be

$$
\frac{\delta \rho}{\rho} \sim \frac{1}{r_{60} M_{P}}
$$

We see that (in agreement with [18]) the requirement of large density perturbations places more severe constraint on the flatness of the inflaton potential than the requirement of successful inflation with 60 e-foldings, which in our scenario requires no fine-tunning. An expected lower limit for $r_{60}$ is around $M_{P f}^{-1}$, which for $M_{P f} \sim \mathrm{TeV}$ would give $\frac{\delta \rho}{\rho} \sim 10^{-15}$. The observed value would require branes with a very short range potential $\left(\sim 10^{4} M_{P}^{-1}\right)$. This would mean that a brane cannot fluctuate at scales $>>10^{-28} \mathrm{~cm}$ and is an extremely rigid object.

There can be other sources of the density fluctuations, e.g. coming from the branes falling on the orientifold plane earlier, 60 e-folds before the end of inflation (see discussion at the end of the previous section). To conclude, the precise origin of the density perturbations in the present context is an open question and requires an additional study.

Another important issue in theories with $\mathrm{TeV}$ scale quantum gravity is baryogenesis. As mentioned above, our scenario gives a natural posibility of releasing the inflaton energy predominantly into the branes. This is a desirable starting point for avoiding the bulk graviton over-production. However, even in such a optimistic case the reheating temperature on the brane cannot be arbitrary high since the bulk gravitons can be produced by braneevaporation [1]. In particular, reheating up to the electroweak temperatures is problematic (at least for small $N$ ) and thus the standard scenarios of baryogenesis is not operative. However, in our case, there are new possible sources: first, since the Hubble parameter is so small, the electroweak transition may in fact coincide with the inflationary one and be non-thermal. This will provide an out-of-equilibrium condition. More importantly, the inflaton can directly decay into the baryons via the baryon-number- and CP-violating $M_{P f^{-}}$ suppressed interactions. Such operators can be harmless for proton decay due to unbroken discrete subgroups of $B$ and $L$ [7] (see [15] for an explicit construction). Thus, in principle at least, the baryon number can be generated with a very low reheating temperature. 


\section{E. Conclusions}

In this paper, we present a novel brane inflationary scenario in theories with low scale $(\mathrm{TeV})$ quantum gravity. This inflationary scenario is natural in the brane world picture. In the classical vacuum, $K$ branes, with positive energy densities, sit on top of an orientifold plane, with negative energy density, so the vacuum energy cancels out. In the early universe, some of the branes "start out" relatively displaced in the extra dimensions. This introduces a vacuum energy, which triggers inflation in the non-compact 3-dimensional space. The number of e-foldings can be very large due to the very weak brane-brane interaction at large distances, which is mainly due to the graviton and other massive bulk mode exchanges. The displacement distances between branes are identified with the vacuum expectation values of scalar fields, which are brane (open string) modes. When branes are close to each other, the potential becomes steep and inflation ends rapidly, releasing energy mostly into radiation on the branes. In this scenario, the electroweak Higgs field may even play the role of the inflaton.

Although this brane inflationary scenario emerges rather naturally in the brane world picture and the overall picture looks promising, its details are quite model-dependent. Precise predictions seem to require a better understanding of the moduli stabilization and supersymmetry breaking mechanism. On the other hand, we can use the inflationary requirements to further constraint the brane world dynamics. For example, the shape of the inflaton potential depends strongly on the masses of the light bulk (closed string) modes, which are massless before moduli stabilization and supersymmetry breaking. They are expected to have tiny masses if supersymmetry breaking occurs on the brane and is then transmitted to the bulk.

Although the brane inflation can easily reheat the brane to a temperatures around the electroweak scale $M_{e w}$, for small number of extra dimensions and low $M_{P f}$, this is forbidden, due to the subsequent evaporation into the bulk gravitons [1]. Thus the standard electroweak baryogenesis cannot be applied in such a case. However, in the brane inflation context, there are new potential sources, which can allow baryon number generation at much lower

temperatures. This is due to the possibility of direct $B$-and $C P$-violating decays of the inflaton into the brane-baryons. It is important to study this issue in greater detail.

\section{ACKNOWLEDGMENTS}

We thank Philip Argyres, Gregory Gabadadze, Zurab Kakushadze, Gary Shiu and Alex Vilenkin for discussions. The research of S.-H.H. Tye is partially supported by the National Science Foundation. 


\section{REFERENCES}

[1] N. Arkani-Hamed, S. Dimopoulos and G. Dvali, Phys. Lett. B429 (1998) 263, hepph/9803315; hep-ph/9807344, Phys. Rev. D (to be published).

[2] J. Lykken, Phys. Rev. D54 (1996) 3693.

[3] E. Witten, Nucl. Phys. B471 (1996) 135.

[4] V.A. Rubakov and M.E. Shaposhnikov, Phys. Lett. B125 (1983) 136; see also, A. Barnaveli and O. Kancheli, Sov. J. Nucl. Phys. 51 (1990) 573.

[5] G. Dvali and M. Shifman, Nucl. Phys. B504 (1997) 127; Phys. Lett. B396 (1997) 64.

[6] J. Polchinski, "String Theory", Cambridge University Press, 1998.

[7] I. Antoniadis, N. Arkani-Hamed, S. Dimopoulos and G. Dvali, Phys. Lett. B436 (1998) 257, hep-ph/9804398.

[8] G. Shiu and S.-H.H. Tye, Phys. Rev. D58 (1998) 106007, hep-th/9805147.

[9] Z. Kakushadze, Phys. Lett. B434 (1998) 269; Nucl. Phys. B535 (1998) 311; Phys. Rev. D58 (1998) 101901;

Z. Kakushadze and S.-H.H. Tye, Phys. Rev. D58 (1998) 126001.

[10] Z. Kakushadze and S.-H.H. Tye, hep-th/9809147.

[11] P.C. Argyres, S. Dimopoulos and J. March-Russell, hep-th/9808138.

[12] G.F. Giudice, R. Rattazzi and J.D. Wells, hep-ph/9811291;

S. Nussinov and R. Shrock, hep-ph/9811323;

E.A. Mirabelli, M. Perelstein and M.E. Peskin, hep-ph/9811337;

T. Han, J.D. Lykken and R.-J. Zhang, hep-ph/9811350;

J.L. Hewett, hep-ph/9811356.

[13] N. Arkani-Hamed and S. Dimopoulos, hep-ph/9811353;

Z. Berezhiani and G. Dvali, hep-ph/9811378.

[14] N. Arkani-Hamed, S. Dimopoulos, G. Dvali and J. March-Russell, hep-ph/9811448;

K.R. Dienes, E. Dudas and T. Gherghetta, hep-ph/9811428.

[15] Z. Kakushadze, hep-ph/9811193; hep-th/9812163.

[16] K.R. Dienes, E. Dudas and T. Gherghetta, Phys. Lett. B436 (1998) 55, hepph/9803466; hep-ph/9806292; hep-ph/9807522.

[17] D. Lyth, hep-ph/9810320.

[18] N. Kaloper and A. Linde, hep-th/9811141.

[19] K.R. Dienes, E. Dudas, T. Gherghetta and A. Riotto, hep-ph/9809406.

[20] A. Riotto and M. Magiore, hep-ph/9811089.

[21] E. Witten, Nucl. Phys. B443 (1995) 85.

[22] R. Sundrum, hep-ph/9805471; hep-ph/9807348.

[23] N. Arkani-Hamed, S. Dimopoulos and J. March-Russell, hep-th/9809124.

[24] I. Antoniadis and C. Bachas, hep-th/9812093.

[25] For a partial list, see, e.g.,

G. Pradisi and A. Sagnotti, Phys. Lett. B216 (1989) 59;

M. Bianchi and A. Sagnotti, Phys. Lett. B247 (1990) 517; Nucl. Phys. B361 (1991) 539 ;

E.G. Gimon and J. Polchinski, Phys. Rev. D54 (1996) 1667;

A. Dabholkar and J. Park, Nucl. Phys. B472 (1996) 207, hep-th/9602030; Nucl. Phys. B477 (1996) 701, hep-th/9604178;

E.G. Gimon and C.V. Johnson, Nucl. Phys. B477 (1996) 715, hep-th/9604129; 
J. Polchinski, Phys. Rev. D55 (1997) 6423, hep-th/9606165;

M. Berkooz and R.G. Leigh, Nucl. Phys. B483 (1997) 187;

C. Angelantonj, M. Bianchi, G. Pradisi, A. Sagnotti and Ya.S. Stanev, Phys. Lett. B385 (1996) 96;

Z. Kakushadze, Nucl. Phys. B512 (1998) 221;

Z. Kakushadze and G. Shiu, Phys. Rev. D56 (1997) 3686; Nucl. Phys. B520 (1998) 75;

J.D. Blum and A. Zaffaroni, Phys. Lett. B387 (1996) 71;

G. Zwart, Nucl. Phys. B526 (1998) 378;

G. Aldazabal, A. Font, L.E. Ibáñez and G. Violero, hep-th/9804026;

Z. Kakushadze, G. Shiu and S.-H.H. Tye, Nucl. Phys. B533 (1998) 25;

R. Blumenhagen and A. Wisskirchen, Phys. Lett. B438 (1998) 52;

L.E. Ibáñez, R. Rabadan and A.M. Uranga, hep-th/9808139.

[26] J. Dai, R.G. Leigh and J. Polchinski, Mod. Phys. Lett. A4 (1989) 2073;

R.G. Leigh, Mod. Phys. Lett. A4 (1989) 2767;

P. Hovara, Nucl. Phys. B327 (1989) 461; J. Polchinski, Phys. Rev. Lett. 75 (1995) 4724.

[27] C. Vafa, Nucl. Phys. B469 (1996) 403;

D. Morrison and C. Vafa, Nucl. Phys. B473 (1996) 74; Nucl. Phys. B476 (1996) 437.

[28] For a review, see e.g., C.P. Bachas, hep-th/9806199.

[29] J.C.Price, in proc. Int. Symp. on Experimental Gravitational Physics, ed. P.F. Michelson, Guangzhou, China (World Scientific, Singapore 1988); J.C. Price et. al., NSF proposal 1996; A. Kapitulnik and T. Kenny, NSF proposal, 1997; J.C. Long, H.W. Chan and J.C. Price, hep-ph/9805217. 